\title{
Understanding the impact of removable partial dentures on patients' lives and their attitudes to oral care
}

\author{
Mita Shaha, ${ }^{\star 1}$ Roshan Varghese ${ }^{2}$ and Mounir Atassi ${ }^{3}$
}

\section{Key points}

The current findings indicate that differences between full and removable partial denture wearers (RPDWs) relate to their remaining natural teeth and their desire to protect these to avoid the need for full dentures.
RPDWs would like more information about how to care for their removable partial dentures and would follow information/advice from their dentist when given.
RPDWs report experiencing real trauma (emotional and physical) from when they lose a tooth and are told they need a partial denture through to getting the new partial denture.

\begin{abstract}
Introduction Due to a rising prevalence of removable partial denture (RPD) use, there is an unmet need to explore the psychological impact on patient wellbeing.
\end{abstract}

Aim To assess the psychological effects of consumers losing teeth and receiving partial dentures to better manage their needs.

Design Four market research surveys, undertaken on behalf of GlaxoSmithKline Consumer Healthcare, are described.

Setting Surveys were conducted across the US, Japan, Malaysia, Colombia, Brazil, Poland and Italy.

Materials and methods Quantitative/qualitative methods were used to gain knowledge regarding patients' experiences living with partial dentures.

Results In a global market research survey involving 3,952 RPD wearers (RPDWs), 54\% said that losing teeth was difficult to accept, $40 \%$ were concerned about their appearance, 32\% were embarrassed about wearing dentures and $62 \%$ wished that more oral care education was available when they were younger. In another survey of $>1,000$ RPDWs, self-esteem was negatively impacted in 51\% of respondents and $57 \%$ reported anxiety around dental visits.

Conclusions Global market research confirmed that RPDWs can have emotional and practical needs that are not well understood/documented by dental healthcare professionals (HCPs). Consumer research can enhance understanding of patients' needs by dental HCPs and provide suggestions about how they may address them.

\section{Introduction}

Removable partial dentures (RPDs) are used to replace missing teeth to improve masticatory efficiency, enhance appearance, prevent unwanted teeth movement and/or improve phonetics. ${ }^{1}$ They may be chosen over fixed prosthetics for various reasons, including financial limitations, to facilitate hygiene

${ }^{1}$ Global Consumer Insights Lead for Denture Care, GSK Consumer Healthcare, St George's Avenue, Weybridge, Surrey, KT13 ODE, UK; ${ }^{2}$ Medical Affairs Director, GSK

Consumer Healthcare, St George's Avenue, Weybridge, Surrey, KT13 ODE, UK; ${ }^{3}$ Principal Medical Affairs Scientist, GSK Consumer Healthcare, St George's Avenue, Weybridge Surrey, KT13 ODE, UK

*Correspondence to: Mita Shaha

Email address: mita.x.shaha@gsk.com

Refereed Paper.

Accepted 21 July 2020

https://doi.org/10.1038/s41415-021-2949-4 access, or to overcome biomechanical issues associated with dental implants. ${ }^{1}$

The number of people with partial dentures is rising in line with increased numbers of partially dentate adults. This is thought to correlate with increased life expectancy and numbers of elders in the population, and a shift from total edentulism towards partial edentulism as oral hygiene improves. ${ }^{1}$ An analysis in the US highlighted the growing need for prosthodontic treatment, expected to exceed the supply available in $2020 .^{2}$ Recent data from a global market research study, conducted in 2015, suggested approximately $19 \%$ of adults wear dentures, of whom most (60\%) were partial denture wearers (Fig. 1). ${ }^{3}$

While more people now wear RPDs, studies indicate poor patient acceptance and an increased incidence of caries and periodontal issues compared with fully dentate individuals. ${ }^{4}$ Understanding the reasons behind poor acceptance/hygiene could help improve outcomes, yet little is known about the psychological aspects of tooth loss and subsequent use of RPDs in daily life. Subsequently, this aspect of patient care is often overlooked.

Exploring the reactions to tooth loss in 150 partially dentate people in the UK and Hong Kong demonstrated that tooth loss can majorly impact the lives of those affected. Half (49\%) of participants experienced difficulty in accepting their partial tooth loss, with many reporting decreased self-confidence, restriction in dietary choice and social activities, and avoidance of close relationships. ${ }^{5}$ Other studies confirmed these results, showing how RPD wearers (RPDWs) experience negative quality of life effects, including avoidance of certain foods, difficulty relaxing, isolation, discomfort, 
nervousness, pain and distress. ${ }^{6}$ Emotions surrounding tooth loss may differ between populations and cultures. In London, people took longer to accept their tooth loss, whereas those in Hong Kong were more affected by food restrictions. ${ }^{5}$

Since patient engagement and motivation is key to treatment success, more knowledge of the patient perspective is important. Considering the emotional and psychological needs of RPDWs by dentists is integral to help patients overcome certain issues and improve treatment outcomes. Market research surveys that collect insights from patients can provide helpful information and are undertaken by healthcare companies. Resulting data complements that from standard clinical/ epidemiological studies by deepening knowledge around patient behaviours, further supporting product innovation and development. Consequently, market research surveys provide critical information that can help inform healthcare professionals (HCPs) about the potential needs of their patients.

This publication reports market research data collected and funded by GlaxoSmithKline Consumer Healthcare (GSKCH) from four surveys among denture wearers. Quantitative and qualitative methods were included; the latter used exploratory, descriptive designs and themed approaches for data analysis. Immersive ethnographic techniques were included to gather comprehensive accounts of different actions, behaviours and beliefs to enhance understanding of social practices. All surveys were conducted on behalf of GSKCH to better understand the needs, motivations and behaviours of people with RPDs. None are in the public domain.

\section{Materials and methods}

Four market research surveys are included in this publication as detailed below (Table 1). These surveys complied with the Market Research Society code of conduct.

Survey one: market research consumer survey and analysis - US, Japan, Malaysia, Colombia, Brazil, Poland and Italy

This survey was conducted on behalf of GSKCH by a global market research company based in the UK in 2015. The research was undertaken in seven countries: the US, Japan, Malaysia, Colombia, Brazil, Poland and Italy, and included $>7,000$ subjects with full or RPDs. Subjects were interviewed (35-40

Fig. 1 Proportion of denture wearers with full, full and partial, and partial dentures. Data collected in a study in 2014 illustrate the large numbers of denture wearers that have partial dentures. ${ }^{3}$ Base: adults $>18$ years of age. $N=1,000$ per country, denture wearers only

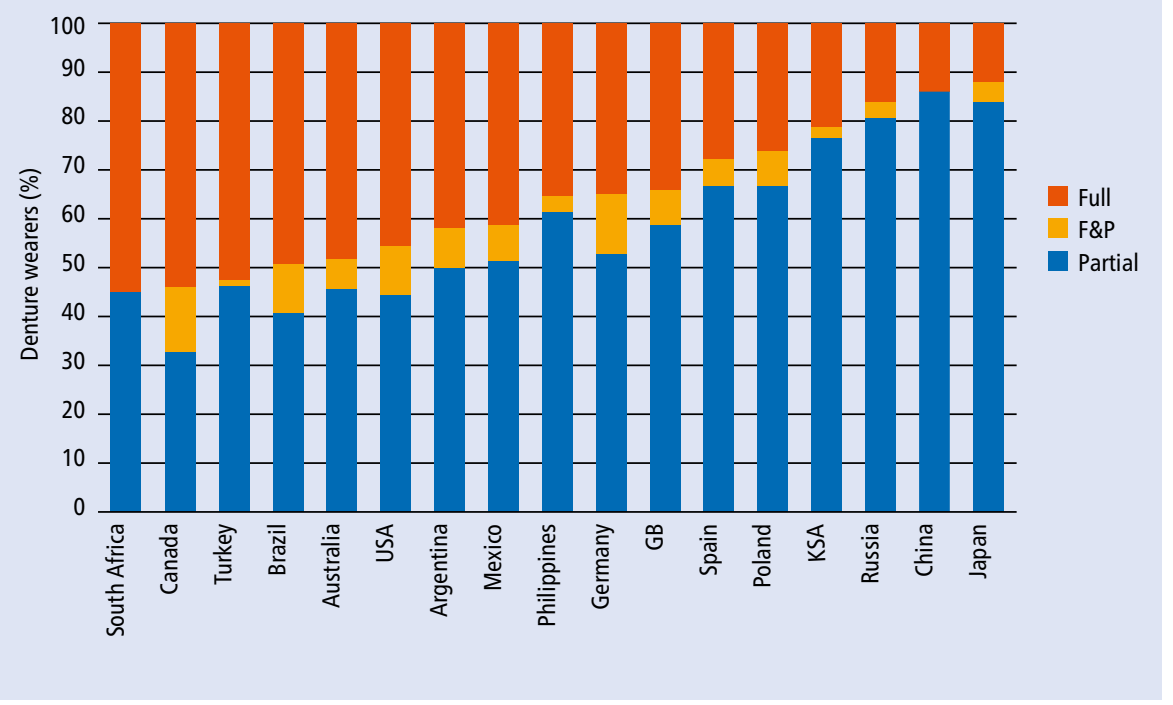

minutes' duration), either online or face to face; all interviews were conducted using the same questionnaire. Online interviews were undertaken among people $40-75$ years of age in the US and Japan, whereas face-to-face interviews were undertaken among people 40-75 years of age in Malaysia, Colombia, Brazil, Poland and Italy.

Interviewees answered survey questions assessing demographics, denture cleansing methods, use/selection of denture cleansing products/adhesives and different questions about aspects of wearing dentures. Data collected from RPDWs and full denture wearers were analysed and compared. The RPDW group included individuals with partial dentures in the upper mouth, lower mouth, or both upper and lower mouth, but excluded subjects with full dentures in either the upper or lower mouth.

\section{Survey two: market research consumer} survey and analysis - US

This national survey was conducted on behalf of GSKCH to gather insights on the emotional and social impact of wearing RPDs, undertaken in the US in 2013. It consisted of an e-mailed invitation, an online screener questionnaire and survey questionnaire, and included 1,039 RPDWs $\geq 18$ years of age.

\section{Survey three: insights research - US and Japan}

Market research was conducted in Japan and the US, involving home observations and in-depth focus group discussions among
Table 1 Outline of market research studies included (RPDs = removable partial dentures, RPDWS = removable partial denture wearers)

\begin{tabular}{l|l|l|l}
$\begin{array}{l}\text { Survey } \\
\text { number }\end{array}$ & $\begin{array}{l}\text { Countries } \\
\text { involved }\end{array}$ & Included & Aims \\
Survey one & $\begin{array}{l}\text { US, Japan, Malaysia, } \\
\text { Colombia, Brazil, } \\
\text { Poland and Italy }\end{array}$ & $\begin{array}{l}>7,000 \text { subjects with } \\
\text { full or RPDs (40-75 } \\
\text { years of age) }\end{array}$ & $\begin{array}{l}\text { To gather data on demographics, methods } \\
\text { of denture cleansing, use and selection of } \\
\text { denture cleansing products and adhesives, } \\
\text { and attitudes to wearing dentures }\end{array}$ \\
\hline Survey two & US & $\begin{array}{l}1,039 \text { RPDWS (18 } \\
\text { years of age and over) }\end{array}$ & $\begin{array}{l}\text { To gather insights on the emotional and } \\
\text { social impact of wearing RPDs }\end{array}$ \\
\hline Survey three & US and Japan & $\begin{array}{l}\text { Male and female RPDs } \\
\text { (40-70 years of age) }\end{array}$ & $\begin{array}{l}\text { To gather insight into personal highs and } \\
\text { lows of partial denture use, including } \\
\text { motivations, problems, issues, frustrations, } \\
\text { misconceptions and fears }\end{array}$ \\
\hline Survey four & Canada and Japan & $\begin{array}{l}60 \text { male and female } \\
\text { RPDWS (30 in Canada, } \\
30 \text { in Japan) }\end{array}$ & $\begin{array}{l}\text { To connect and map consumer responses } \\
\text { across multiple different points of contact }\end{array}$ \\
\hline
\end{tabular}


RPDWs in 2017. Participants were screened and selected by recruitment agencies on behalf of GSKCH. All had worn partial dentures for either one, three or five years. In total, ten consumers 40-70 years of age were included in Tokyo, Japan and ten consumers 40-65 years of age in New York, US.

A discussion guide was constructed to ensure broad areas of interest were covered. Discussions were designed to gather personal insights into the highs and lows of partial denture use, including motivations, issues, frustrations, misconceptions and fears.

\section{Survey four: insights research - Canada and Japan}

This research involved focus groups and was undertaken in Canada and Japan in 20152016. It aimed to connect and map consumer responses across multiple different points of contact.

The focus groups were held among RPDWs who were enrolled based on their response to a recruitment questionnaire to ensure articulate participants. The discussions were designed to be interactive and were directed by a skilled strategic moderator. Overall, 60 candidates were included - 30 in Canada and 30 in Japan.

Four four-hour workshops were held in Canada and Japan. Two focus groups were held with RPDWs who had worn dentures for up to two years and two with RPDWs with $>2$ years of denture wear.

\section{Results}

In total, 7,367 subjects were included in survey one; 3,952 were RPDWs and 3,415 were classed as full or full-plus-partial denture wearers. Overall, demographics were similar between partial and full denture wearers; most participants were $>50$ years of age, with an equal gender split (Table 2). RPDWs' dental conditions related mainly to their remaining natural teeth; for example, plaque, tooth decay and receding gums. The desire to preserve/protect remaining natural teeth was an overarching motivator for RPDWs. Receding gums were a more prominent concern in the US and Japan, although it was unclear if this difference may have reflected greater awareness of gum disease in these markets.

In survey one, over half (54\%) of RPDWs said that losing teeth was difficult to accept. Data indicate that oral care for RPDWs is

Table 2 Demographics and dental history data from survey one

\begin{tabular}{l|l|l} 
Characteristics & $\begin{array}{l}\text { Partial denture } \\
\text { wearers } \\
(n=3,952)\end{array}$ & $\begin{array}{l}\text { Full denture } \\
\text { wearers } \\
(n=3,415)\end{array}$ \\
\hline Mean age (years) & 57 & 61 \\
\hline Age & &
\end{tabular}

\begin{tabular}{l|l|l}
\hline Age group (\%) & 11 & 4 \\
\hline 40 to 44 years & 16 & 9 \\
\hline 45 to 49 years & 13 & 9 \\
\hline 50 to 54 years & 21 & 18 \\
\hline 55 to 59 years & 12 & 16 \\
\hline 60 to 64 years & 25 & 37 \\
\hline 65 to 73 years & 2 & 6 \\
\hline 74 to 75 years & & 61 \\
\hline
\end{tabular}

Gender (\%)

\begin{tabular}{l|l|l}
\hline Male & 51 & 53 \\
\hline Female & 49 & 47 \\
\hline
\end{tabular}

Dentures used (\%)

\begin{tabular}{l|l|l}
\hline Full upper & - & 90 \\
\hline Full lower & - & 46 \\
\hline Partial upper denture or removable bridge & 77 & 2 \\
\hline Partial lower denture or removable bridge & 43 & 12 \\
\hline Mean duration of denture use (years) & &
\end{tabular}

Mean duration of denture use (years)

\begin{tabular}{l|l|l}
\hline Full upper & 7 & 8 \\
\hline Full lower & 6 & 8 \\
\hline Partial upper denture or removable bridge & - & 7 \\
\hline Partial lower denture or removable bridge & - & 8 \\
\hline Mean age at first denture (years) & 51 & 54 \\
\hline
\end{tabular}

\begin{tabular}{l|l|l}
\hline Main cause of tooth loss [multiple answers possible] (\%) \\
\hline Decay/infection & 41 & 47 \\
\hline Gum disease & 19 & 30 \\
\hline Limited dental visits from young age & 24 & 30 \\
\hline
\end{tabular}

focused on preventing further tooth loss, with $76 \%$ of respondents agreeing with the statement: 'When I clean my teeth, I am mainly trying to prevent any tooth loss/ further tooth loss'.

Although $42 \%$ of RPDWs said they avoid trips to the dentist unless essential, RPDWs are more likely to visit the dentist than full denture wearers due to fear of further tooth loss $(27 \%$ of RPDWs visit every six months compared with $17 \%$ of full denture wearers). More RPDWs than full denture wearers (59\% vs $51 \%$ ) admitted to asking their dentist for advice and recommendations, but fewer RPDWs used specialist denture care products (denture cleansers and adhesives). Twenty percent of RPDWs versus $41 \%$ of full denture wearers had used a denture adhesive in the past three months; $55 \%$ of these RPDWs felt adhesives were unnecessary because they only had partial dentures. Similarly, for the use of cleaners, $39 \%$ of partial denture wearers versus $46 \%$ of full wearers had used a denture cleanser in the past three months. Many RPDWs believed their oral care routine was sufficient (59\%) and that regular toothpaste was fine for 
their natural teeth and RPDs (54\%), and that cleansers were unnecessary because they only wore partial dentures (44\%).

Survey two highlighted inadequate understanding regarding optimal care for RPDs. Respondents were only partially aware of proper denture care regimens - almost one in five (13\%) reported rarely following a set cleaning routine for their RPDs and more than a third (34\%) usually leave their partial dentures in their mouth for 16 hours or longer. One in four (25\%) confessed to often wearing RPDs when they sleep.

Survey three revealed that RPDWs want to prevent further tooth loss and feel that oral care to help prevent this should be easy. Currently, it is not perceived as easy, but rather as cumbersome, messy and infuriating. RPDWs reported having partial dentures complicates their normal oral care regime (Box 1) and stressed a lack of recommended guidelines.

Survey four suggested RPDWs take steps to improve their oral care regime after losing teeth. All undertake at least two additional steps compared with their pre-denture routine to protect their natural teeth from further loss. This intense focus on oral care underscores a fear that they are not doing enough, and despite their best efforts, may yet lose more teeth.

\section{Attitudes, anxieties and stigmas - all surveys}

Different attitudes between full and partial denture wearers in relation to life and health were shown in survey one. Partial wearers experienced more anxiety and guilt than full denture wearers about their oral hygiene (Fig. 2).

Forty percent of RPDWs reported anxiety around appearance, $33 \%$ expressed embarrassment and $62 \%$ wished they were more educated about the importance of oral care when younger. They didn't wish to be defined by wearing dentures.

In survey two, $51 \%$ of RPDWs stated their confidence/self-esteem was negatively impacted and a similar proportion agreed that losing teeth motivated them to improve oral care. Most respondents (79\%) said they feel more informed about caring for their existing teeth than partial dentures. Over half (57\%) reported fear around visiting a dentist in case they might lose more teeth.

Insight research in survey three revealed that RPDWs often have a strong sense of inferiority resulting from tooth loss and denture use. They felt that being a partial denture wearer is taboo
Box 1 Typical oral care habits reported by US and Japanese partial wearers show multiple cumbersome steps with many pain points throughout their daily routine

1. Brush teeth in the morning (most use mainstream toothpaste), some use sensitivity toothpaste (some skip this step and go straight to 2)

2. A few then use mouthwash - gargling for a while then spitting out into sink

3. Remove partial from the overnight storage cup/glass where it has been sitting in antibacterial cleansing fluid (or bicarbonate of soda) (note some do step 3 and 4, before 1)

4. Rinse partial under tap water

5. Minority apply adhesive to partial (tends to be those later in their journey)

6. Put partial in mouth (for those with adhesive - they wait a while [can be up to 30 minutes] until the partial feels secure)

7. Some eat breakfast then if time allows, repeat steps 1 and 2 with their partial in their mouth (for those that suffer food or partial irritability at this stage, they are compelled to remove their partial and repeat step 4-6). and not overtly spoken about; such feelings were exacerbated by the wider stereotype of denture wearers being old and mocked on social media.

Interestingly, many RPDWs did not accept that they were a denture wearer and did not want to be treated as one.

Across Canada and Japan in survey four, the discussion revealed that all RPDWs have worked to accept their partial dentures as their new normal and move beyond the shame of losing teeth.

Reminding participants of the trauma of wearing partial dentures resulted in negative responses; new RPDWs who were still trying to move on said they were jarred by being forced to recall losing teeth. Longer-term RPDWs who had already moved on said they felt resentful at having to revisit something they feel they have put behind them.

\section{Mindsets and types - survey three}

Insight research provided detail on the mindsets of RPDWs. Research undertaken in survey three suggested the length of time the individual had been wearing dentures could allow for subdivision into three mindsets: new partial denture wearers had a reactive mindset and were actively seeking advice; wearers of $>2$ years appeared more proactive and were embedding new habits; whereas RPDWs of $>4$ years were more experienced and accepted partial dentures as part of life, being less reliant on expert advice (Fig. 3).

\section{Fig. 2 Full and partial denture wearers' attitudes to dentures - survey one. Market research data illustrate differences between full and RPDWs in terms of attitudes to life and health}

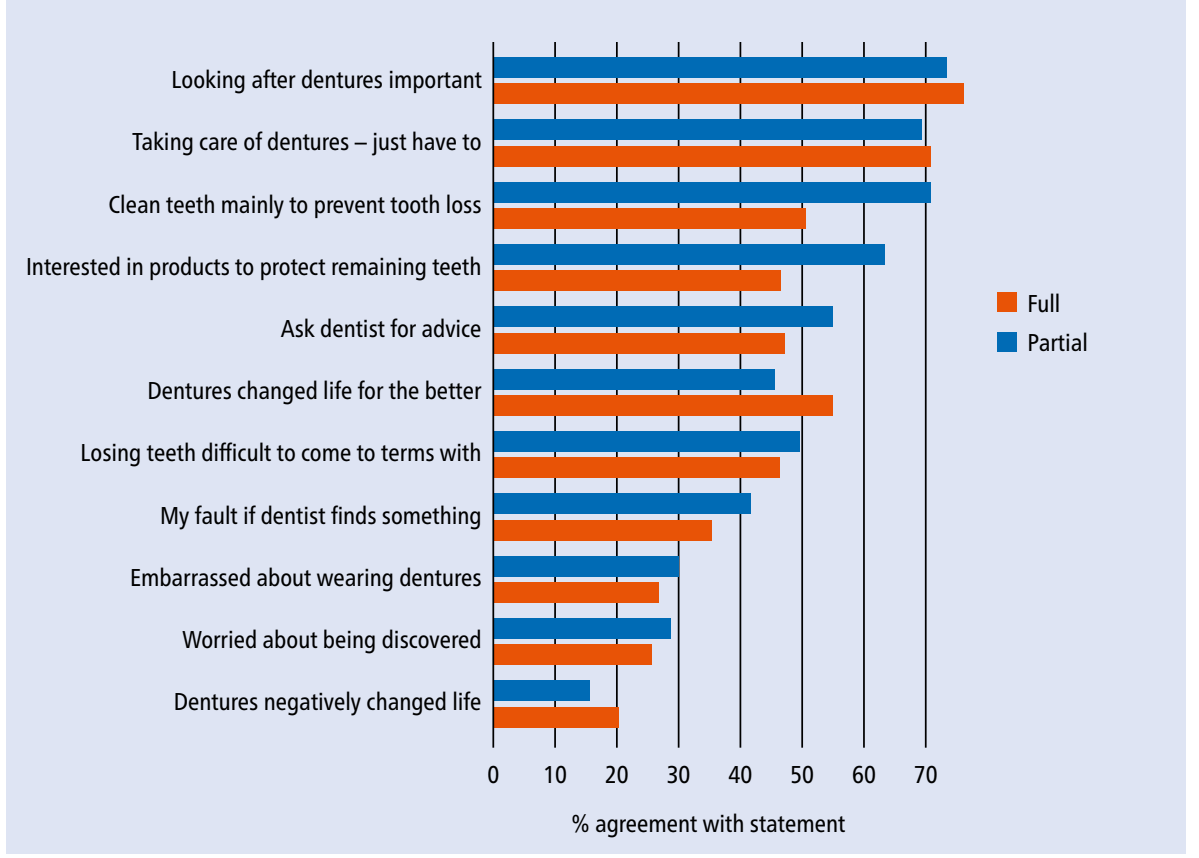


Fig. 3 Mindsets of RPDWs based on duration of denture wearing - survey three. Classing RPDWs by duration of denture wear can inform understanding of how individuals are living with their partials and appropriate approaches for support from dentists

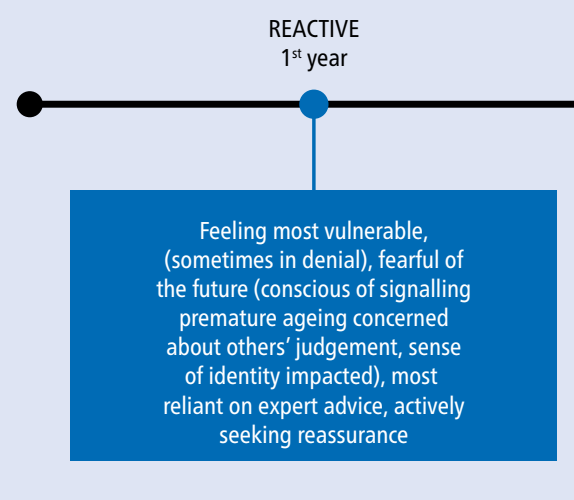

REACTIVE

$2-3+$ years

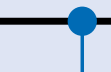

RESISTANT/INERTIA 4-5+ years

\section{Discussion}

This manuscript presents data from a detailed survey among full and RPDWs across seven countries (survey one) and another survey in $>1,000$ RPDWs in the US (survey two), together with results from qualitative interviews and focus discussions with RPDWs, which closely analysed attitudes and emotions (surveys three and four). These descriptive market research studies aimed to identify the emotional, rational and psychological needs of RPDWs to inform product development within GSKCH; however, wider dissemination of data may also enhance understanding of RPDWs by professionals involved in their care.

Overall, these data demonstrate that RPDWs share similar oral hygiene concerns as full denture wearers. Differences for RPDWs relate to their remaining natural teeth and their desire to protect these to avoid the need for full dentures. They report embarrassment and stigma about their partial dentures and wish to avoid further tooth loss. RPDWs are generally younger than full denture wearers and appear willing to change their oral care routine to protect their remaining natural teeth.

While RPDWs do not tend to use, or feel a need for, specialist denture/oral care products, many responses indicate that RPDWs desire more care information for their partials and are receptive to advice from their dentist. Many responders discussed oral care for their whole mouth, suggesting a need for advice on how to care for remaining teeth as well as partial dentures. RPDWs agreed that losing teeth has led to better care of remaining teeth. However, data suggest that many fail to implement the same standard of care with their partial dentures and that many are unaware that proper partial denture care is an important part of a good oral healthcare routine.

A similar lack of knowledge surrounding denture hygiene was demonstrated in an audit to establish deficiencies in patient knowledge surrounding denture hygiene of RPDWs in the UK. ${ }^{7}$ Results showed an association between cleanliness levels of participants' partial dentures and the form in which advice was provided surrounding denture care. The study highlighted the need for improved health education for RPDWs and an increased awareness by the whole dental team. Authors suggested that all RPDWs should receive information and regular reinforcement of key oral hygiene messages.

There is a need to educate patients about oral health issues associated with RPDs. A literature review provided evidence that RPDs increase plaque accumulation and gingivitis, and increase the risk of caries. ${ }^{8}$ Several studies showed that mutans streptococci increase following insertion of an RPD. Thus, the expectation is that all RPDWs are recommended to enrol in regular recall/ maintenance programmes and that dental teams be vigilant to observe early signs of periodontitis or caries, to avoid further tooth loss in patients with existing depleted dentition. ${ }^{8}$

Insights into patients' mindsets and/or need-states may inform dentists to identify different oral health needs across patients and determine how best to approach specific care issues with individual patients. A patient with partial dentures fitted in the last 12 months may need more advice than an RPDW who has had dentures for more than four years. The sense that patients are more psychologically affected at the start of their partial denture-wearing journey suggests that early support by dentists could really help to reassure patients, making them feel more confident. Indeed, patients' motivation for denture treatment should ideally be evaluated before fitting dentures, thus allowing patients to gain realistic expectations of what is possible and allowing dentists to understand a patient's needs. ${ }^{9}$

Collectively, these data may give dental professionals a more detailed impression of emotional and psychological aspects of losing teeth and wearing RPDs, by highlighting attitudes and needs of RPDWs. The findings present a picture of what living with partial dentures is like and how embarrassed RPDWs feel about wearing dentures, as well as identifying potential unmet needs; they can be used to develop tailored strategies for care that address problems that may be specific to particular patients.

Given the likelihood of greater partial denture provision in the future, dental professionals require greater awareness of the oral hygiene of their patients. The wider dental team can also support and reinforce the importance of continuing plaque control and denture care in maintaining good oral health. ${ }^{10}$ Currently, there is limited internationally accepted guidance for RPDWs and HCPs who treat these patients. Academia and industry share a joint responsibility to provide guidance and increase awareness, especially for patient education and product development that is tailored to RPDWs' needs.

The value of consumer insight research in understanding consumer behaviour has been demonstrated in consumer goods and other industries. ${ }^{11,12}$ Further to identifying triggers and barriers for product purchase, they also offer perspective around the target consumer. The value of this research is well established in 
oral health, with previous studies identifying consumer behaviour with a view to enhancing dental professionals' understanding around their patients. ${ }^{13}$

\section{Conclusions}

Market research and insights data from companies such as GSKCH can provide valuable information for dental HCPs. The findings expose the emotional and functional needs of RPDWs and raise a call to action to the profession around providing advice and support that could positively impact these patients' lives.

\section{Conflict of interest}

All market research mentioned in the manuscript was funded by GSK Consumer Healthcare.
All authors are employees of GSK Consumer Healthcare.

\section{Acknowledgements}

Editorial assistance with the preparation of manuscript drafts was provided by Clare Gurton of Vivid Medical Communications Ltd, Loudwater, UK, funded by GSK Consumer Healthcare.

\section{References}

1. Campbell S D, Cooper L, Craddock H et al. Removable partial dentures: The clinical need for innovation. J Prosthet Dent 2017; 118: 273-280.

2. Douglass C W, Watson A J. Future needs for fixed and removable partial dentures in the United States. J Prosthet Dent 2002; 87: 9-14.

3. Data on file. Oral Care Omnibus Study. 2014. (Internal market research report).

4. Allen P F, Jepson N J, Doughty J, Bond S. Attitudes and practice in the provision of removable partial dentures. BrDent J 2008; 204: E2.

5. Fiske J, Davis D M, Leung K C, McMillan A S, Scott S J. The emotional effects of tooth loss in partially dentate people attending prosthodontic clinics in dental schools in England, Scotland and Hong Kong: a preliminary investigation. Int Dent J 2001; 51: 457-462.

6. Jones J A, Omer M B, Spiro A, Kressin N R. Tooth loss and dentures: patients' perspectives. Int Dent J 2003; 53(5 Suppl): 327-334.

7. Milward P, Katechia D, Morgan M Z. Knowledge of removable partial denture wearers on denture hygiene. BrDent J 2013; 215: E20.

8. Preshaw P M, Walls A W, Jakubovics N S, Moynihan P J Jepson $N$ J, Loewy Z. Association of removable partial denture use with oral and systemic health. J Dent 2011; 39: 711-719.

9. Roessler D M. Complete denture success for patients and dentists. Int Dent J 2003; 53(5 Suppl): 340-345

10. Johnson T. Summary of: Knowledge of removable partial denture wearers on denture hygiene. Br Dent 2013; 215: 516-517.

11. Prentice C, Handsjuk N. Insights into Vodka consumer attitude and purchasing behaviours. J Retail Consum Serv 2016; 32: 7-14.

12. Birch $D$, Skallerud $K$, Paul N A. Who are the future seaweed consumers in a Western society? Insights from Australia. Br Food J 2019; 121: 603-615.

13. Axe A S, Varghese R, Bosma M, Kitson N, Bradshaw D J. Dental health professional recommendation and consumer habits in denture cleansing. J Prosthet Dent 2016; 115: 183-188. or other third party material in this article are included in the article's Creative Commons licence, unless indicated otherwise in a credit line to the material. If material is not included in the article's Creative Commons licence and your intended use is not permitted by statutory regulation or exceeds the permitted use, you will need to obtain permission directly from the copyright holder. To view a copy of this licence, visit http://creativecommons.org/licenses/by/4.0.

(c) The Author(s), under exclusive licence to British Dental Association 2021 